Braz J Med Biol Res, January 2012, Volume 45(1) 25-32

doi:10.1590/S0100-879X2011007500159

\section{Effect of thymosin alpha-1 on subpopulations of Th1, Th2, Th17, and regulatory $\mathrm{T}$ cells (Tregs) in vitro}

Xia Yang, Feng Qian, Hai-Yang He, Kai-Jun Liu, Yuan-Zhi Lan, Bing Ni, Yi Tian, Xiao-Lan Fu, Ji Zhang, Zi-Gang Shen, Jian Li, Yi Yin, Jin-Tao Li and Yu-Zhang Wu

The Brazilian Journal of Medical and Biological Research is partially financed by

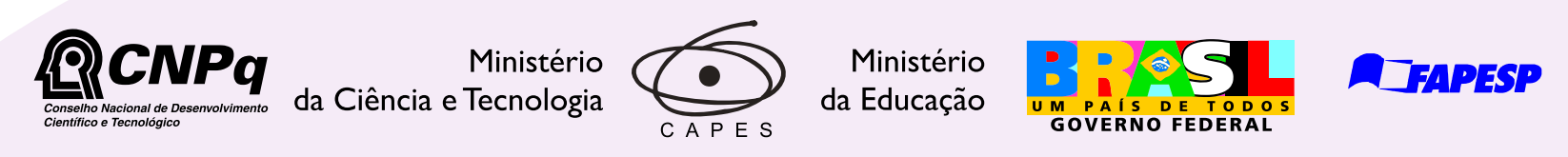

Institutional Sponsors
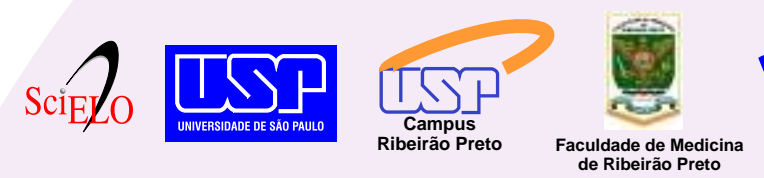

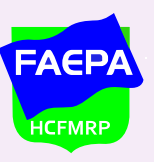

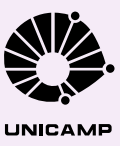

SHIMADZU

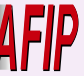

Associaçäo
Fundo de Incentivo
à Pesquisa

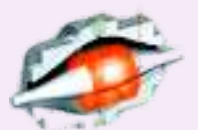

UNICAMP

lore High - Performance MS Orbitrap Technology analitica Thermo 


\title{
Effect of thymosin alpha-1 on subpopulations of Th1, Th2, Th17, and regulatory $T$ cells (Tregs) in vitro
}

\author{
Xia Yang ${ }^{1 *}$, Feng Qiann ${ }^{2 *}$, Hai-Yang He ${ }^{1}$, Kai-Jun Liu ${ }^{1}$, Yuan-Zhi Lan², \\ Bing Ni ${ }^{1}$, Yi Tian ${ }^{1}$, Xiao-Lan Fuํㄹ , Ji Zhang ${ }^{1}$, Zi-Gang Shen ${ }^{1}$, \\ Jian $\mathrm{Li}^{1}$, Yi Yin ${ }^{1}$, Jin-Tao $\mathrm{Li}^{1}$ and $\mathrm{Yu}$-Zhang $\mathrm{Wu}^{1}$ \\ ${ }^{1}$ Institute of Immunology, Third Military Medical University, Chongqing, PR China \\ 2Department of General Surgery, Southwest Hospital, Third Military Medical University, Chongqing, PR China
}

\begin{abstract}
Thymosin alpha $1(\mathrm{Ta} 1)$ has been shown to have beneficial effects on numerous immune system parameters, but little is known about the effects of Ta1 on patients with gastric carcinoma. The objective of this study was to determine the effect of Ta1 on subpopulations of Th1, Th2, Th17, and regulatory T cells (Tregs) in vitro, and to evaluate its efficacy as an immunoregulatory factor in patients with gastric carcinoma. We compared the effect of Ta1 on the frequency of CD4 ${ }^{+}$and CD8 ${ }^{+} \mathrm{T}$ cells, especially the CD $4^{+} \mathrm{CD} 25^{+}$Foxp3 ${ }^{+}$Tregs in peripheral blood mononuclear cells (PBMCs) from gastric carcinoma patients $(\mathrm{N}=35)$ and healthy donors $(\mathrm{N}=22)$. We also analyzed the changes in the proliferation of PBMCs in response to treatment with Ta1, and examined the production of Th1, Th2, and Th17 cytokines by PBMCs and tumor-infiltrating lymphocytes. The treatment of PBMCs from gastric cancer patients, with Ta1 $(50 \mu \mathrm{g} / \mathrm{mL})$ alone increased the percentage of CD4+CD25+Foxp3+ (suppressive antitumor-specific Tregs) from $1.68 \pm 0.697$ to $2.19 \pm 0.795 \%(P<0.05)$. Our results indicate that Ta1 increases the percentage of Tregs and IL-1 $\beta$, TNF- $\alpha$, and IL-6 in vitro.
\end{abstract}

Key words: Ta1; Gastric carcinoma; Tregs; Th1; Th2; Th17

\section{Introduction}

Gastric cancer is the most lethal among malignant diseases worldwide. Over half of gastric cancer cases are reported to occur in developing countries, especially in China (1). Gastric cancers are highly resistant to chemotherapy and radiotherapy; the most common intervention is surgical resection of the tumor with concomitant lymphadenectomy. Regrettably, many clinical studies have shown that surgical resection is associated with an increased risk of recurrence, postoperative complications, and mortality (2). As such, the outcome of surgery for gastric cancer remains very poor, and clinical research has focused on developing more effective adjunct therapies. Some efforts have been made to identify enhanced chemotherapeutic agents to improve the patient's quality of life, to extend survival, and to decrease the duration of illness while the patient reaches remission. Recently, a promising new series of chemotherapeutic agents, including various platinum compounds, fluoropyrimidine derivatives, and S-1 (tegafur, 5-chloro-2,4-dihydropyrimidine, and potassium oxonate) (3), have been described. However, these remain to be fully characterized and clinically validated. There remains a need to seek innovative therapies to treat and resolve gastric cancer.

The cytotoxic action of host $\mathrm{T}$ and natural killer (NK) cells suggests that these cells may inherently harbor effective antitumor properties. However, tumors employ a wide variety of evasive mechanisms to fight the antitumor immune responses mounted by the host (4-6), so that an effective immune response is down-regulated and tolerance to the tumor is established. Among the mechanisms used by tumors to escape host immunity are the production of cytokines such as TGF- $\beta(7)$, IL-10 $(6,8), \mathrm{IL}-17(9,10)$, and perturbation or destruction of immune cells. In addition, recent study has implicated an immunopathological role of $\mathrm{CD} 4{ }^{+} \mathrm{CD} 25^{+}$regulatory $\mathrm{T}$ cells (Tregs) during tumor-medi-

Correspondence: Jin-Tao Li and/or Yu-Zhang Wu, Institute of Immunology, Third Military Medical University, 30 Gaotanyan Street, Shapingba District, Chongqing 400038, China. E-mail: ljtqms@yahoo.com.cn and/or wuyuzhang20006@sohu.com

*These authors contributed equally to this study.

Received June 21, 2011. Accepted November 16, 2011. Available online December 2, 2011. Published January 16, 2012. 
ated anti-host defense $(11,12)$, leading to tumor tolerance. Therefore, the objective of most cancer immunotherapies is to provide essential immunogenic elements to boost tumor-specific immunity and to eliminate those elements that lead to tumor tolerance.

Certain biological response modifiers (BRMs) are thought to be essential for the induction of protective and therapeutic antitumor immunity. Thymosin alpha 1 (Ta1) was described in the 1970 s as a clinically applicable BRM for the treatment of immunodeficiencies and cancers. Ta1 is a polypeptide hormone with immunoregulatory properties that is expressed by various tissues and organs of mammals. Consisting of 28-amino acid residues that are $\mathrm{N}$-terminally acetylated, Ta1 is proteolytically processed from prothymosin a (13). Ta1 has been shown to have beneficial effects on numerous immune system parameters (14), including increasing the antitumor activity of dendritic cells (15), and macrophages (16). Today, Ta1 is being tested in late-stage clinical trials for the treatment of melanoma (17). Although the preliminary results from the clinical trials appear to be promising, little is known about the effects of Ta1 on the proliferation of suppressive antitumor-specific Tregs in patients with gastric carcinoma.

In this study, we investigated the ability of Ta1 to modify various immune cell subpopulations in peripheral blood mononuclear cells (PBMCs) and tumor-infiltrating lymphocytes (TILs) from patients with gastric carcinoma. We compared the effect of Ta1 on the frequency of CD4 ${ }^{+}$ and $\mathrm{CD} 8^{+} \mathrm{T}$ cells, especially the $\mathrm{CD} 4^{+} \mathrm{CD} 25^{+}$Foxp3 ${ }^{+}$Tregs found in PBMCs from gastric carcinoma patients and healthy donors. Moreover, we analyzed the changes in the proliferation of PBMCs in response to treatment with Ta1. Finally, we examined the production of Th1, Th2, and Th17 cytokines by PBMCs and TILs.

\section{Material and Methods}

\section{Patients}

PBMCs or TILs were studied in a group of 35 adult patients (19 females and 16 males) aged 22-70 years (average age: $47.6 \pm 11.2$ ) diagnosed with gastric carcinoma. A donor control group consisted of 22 healthy subjects (12 females and 10 males) aged 23-68 years (average age: $41.5 \pm 10.4)$. The pathological characteristics of all the patients were analyzed by the Department of Pathology (Southwest Hospital, Chongqing, China) between 2008 and 2010, which included classifying their histological subtype and staging their tumor-node-metastasis (TNM). The protocol was approved by the Institutional Ethics Board of Southwest Hospital, Third Military Medical University, and written informed consent was obtained from all patients prior to enrollment.

\section{PBMC isolation \\ PBMCs were isolated according to manufacturer}

instructions by centrifugation at $1500 \mathrm{~g}$ for $20 \mathrm{~min}$ on a Ficoll-Hypaque gradient (Sigma, USA). The phase containing the white blood cells was retained and washed three times with RPMI 1640 medium supplemented with $10 \%$ heat-inactivated fetal calf serum (Gibco Invitrogen, Italy). The PBMCs were diluted to $1 \times 10^{6}$ cells $/ \mathrm{mL}$ for analysis.

\section{TIL isolation}

TILs were isolated from tumor tissues by enzymatic digestion as previously described (18). In brief, after surgical resection, the tissues maintained under sterile conditions were finely minced and enzymatically digested in RPMI $1640 \mathrm{U} / \mathrm{mL}$ hyaluronidase V, $30 \mathrm{U} / \mathrm{mL}$ DNase I, $300 \mathrm{U} / \mathrm{mL}$ collagenase type IV (all from Sigma), and antibiotics (100 $\mathrm{U} / \mathrm{mL}$ penicillin, $100 \mu \mathrm{g} / \mathrm{mL}$ streptomycin, and $100 \mu \mathrm{g} / \mathrm{mL}$ gentamycin, all from Life Technologies, USA). After digestion for 3-4 $\mathrm{h}$ at room temperature, the suspension was filtered through a $25-\mu \mathrm{m}$ nylon filter. The filtrate was separated on a Ficoll-Hypaque gradient to isolate the TILs and tumor cells, after which the cells were washed three times with PBS supplemented with 5\% FBS (19).

\section{Cell culture}

The PBMCs and TILs were treated with various concentrations of Ta1 as described (20). Briefly, the cells were incubated with RPMI 1640 medium supplemented with $10 \%$ heat-inactivated fetal calf serum and recombinant human IL-2 (100 U/mL) in 96-well, round-bottom plates at $37^{\circ} \mathrm{C}$ in an atmosphere of $5 \% \mathrm{CO}_{2}$ for $72 \mathrm{~h}$. The PBMCs obtained from patients were cultured with or without Ta1 as detailed below. Cell yield and viability were evaluated using the Trypan blue dye exclusion method. Cells were then harvested and the supernatants were collected and stored at $-80^{\circ} \mathrm{C}$ for cytokine analysis.

\section{Flow cytometry}

All antibodies, the fixation/permeabilization concentrate and diluent, and the permeabilization buffer were purchased from eBiosciences (eBioscience, Inc., USA). After culturing for $72 \mathrm{~h}$, the cells were collected and washed twice with PBS. The cells were stained with PE-Cy5.5-conjugated antihuman CD4 monoclonal antibody (mAb), FITC-conjugated anti-human CD8 mAb, and PE-conjugated anti-human CD25 mAb or the appropriate isotype control mAbs in 100 $\mu \mathrm{L}$ PBS for $45 \mathrm{~min}$ at $4^{\circ} \mathrm{C}$. These antibodies were used at a concentration of $20 \mu \mathrm{L}(0.125 \mu \mathrm{g})$ per $10^{6}$ cells in a total staining volume of $100 \mu \mathrm{L}$. Positive controls for APC, FITC, PE-Cy5.5, and PE staining were also included in the analysis. The cells were then washed twice with PBS, and subjected to intracellular staining using APC-conjugated anti-human Foxp3 mAb according to manufacturer instructions. The antibody was used at a concentration of $20 \mu \mathrm{L}$ $(0.125 \mu \mathrm{g})$ per $10^{6}$ cells in a $100-\mu \mathrm{L}$ total staining volume. The cells were then resuspended in permeabilization buffer and analyzed by flow cytometry on a BD FACSAria instrument 
(BD Biosciences, USA) using the Cell Quest software.

Cytokine secretion in the culture supernatants was assessed with a human Th1/Th2 11plex multiplex kit and a human IL-17A simplex kit (both from Bender MedSystems, Austria) according to manufacturer instructions. The concentrations of IL-1 $\beta$, IL-2, IL-4, IL-5, IL-6, IL-10, IL-12, IFN- $\gamma$, TNF- $\alpha$, TNF- $\beta$, and IL-17A were determined by flow cytometry. Briefly, $25 \mu \mathrm{L}$ culture supernatants or cytokine standards was aliquoted into tubes, and multiplex antibody beads were added to each sample. For detection, biotinconjugated multiplex antibodies were added to the samples, and these were incubated at room temperature and in the dark for $2 \mathrm{~h}$ and then washed to remove unbound proteins. The samples were incubated at room temperature in the dark for $1 \mathrm{~h}$ with streptavidin-PE solution. After washing, the samples were resuspended in PBS and 10,000 events were counted per sample for measurement by flow cytometry on a BD FACSAria instrument (Becton, Dickinson and Company, USA). The data for each cytokine were acquired using the FlowCytomix Software (Bender MedSystems).

\section{$\left[{ }^{3} \mathrm{H}\right]$-thymidine analysis}

Sixteen hours before collecting the cells, $1 \mu \mathrm{Ci}\left[{ }^{3} \mathrm{H}\right]-$ thymidine was added to each well and adequately mixed. The radioactivity emitted by the cells in the individual wells was measured using an LS 6500 liquid scintillation counter (Beckman) and was reported as counts per min (cpm).

\section{Statistical analysis}

Data are reported as means \pm SD and were analyzed statistically by the Student $t$-test (SPSS 13.0, SPSS Inc., USA). $P<0.05$ was considered to be statistically significant.

\section{Results}

Ta1 promotes the generation of $\mathrm{CD} 4^{+} \mathrm{CD} 25^{+} \mathrm{Foxp} 3^{+}$ (Tregs) from PBMCs. We further investigated the effect of Ta1 on Tregs, which prevent host antitumor immune responses. The PBMCs from patients with gastric cancer and healthy donors were co-cultured in Ta1-containing medium. The frequencies of Tregs were determined by measurement of $\mathrm{CD}^{+}, \mathrm{CD}^{2} 5^{+}$, and Foxp $3^{+}$molecular markers, which are most commonly associated with Treg phenotypes. There was no significant change in the percentage of CD4 in PBMCs isolated from healthy people and cancer patients in the presence or absence of Ta1 $(P>0.05$; data not shown). As shown in Figure $1 \mathrm{~A}, \mathrm{CD} 4^{+} \mathrm{CD} 25^{+} \mathrm{Foxp} 3^{+}$levels in Ta1-treated PBMCs from healthy donors were higher than that in Ta1-untreated PBMCs. The mean percentage of $\mathrm{CD} 4^{+} \mathrm{CD} 25^{+}$Foxp $3^{+}$Tregs in PBMCs from healthy donors was found to be increased from $1.45 \pm 0.638 \%$ under PBS treatment to $2.22 \pm 0.401 \%$ under Ta $1(50 \mu \mathrm{g} / \mathrm{mL})$ treatment $(P<0.05)$. As shown in Figure 1B, the mean percentage of $\mathrm{CD} 4^{+} \mathrm{CD} 25^{+} \mathrm{Foxp}^{+}$Tregs in PBMCs from patients with gastric cancer was statistically increased from $1.68 \pm 0.697 \%$ under PBS treatment to $2.19 \pm 0.795 \%$ under Ta1 $(50 \mu \mathrm{g} /$ $\mathrm{mL})$ treatment $(P<0.05)$. No significant differences were observed either at baseline or in the changes of HIV patients induced by Ta1 treatment, in terms of CD4 and CD8 lymphocyte subset changes at week 12 (21). These results indicate that Ta1 treatment can induce CD $4^{+} \mathrm{CD} 25^{+} \mathrm{Foxp} 3^{+}$ lymphocyte expansion, suggesting that the Ta1-induced Treg expression subsequently could hinder the antitumor action mediated by the host immune response. The effect of Ta1 on the expression of CD $4^{+} \mathrm{CD} 25^{+} \mathrm{Foxp} 3^{+}$Tregs has not been reported.

Moreover, the $\mathrm{CD}^{+}$and Foxp3 ${ }^{+}$markers are known to be involved in another Treg $\left(\mathrm{CD}^{+} \mathrm{Foxp} 3^{+}\right)$subpopulation. In our analysis, CD8 ${ }^{+} \mathrm{Foxp}^{+} \mathrm{T}$ cells did not exhibit any statistically significant changes in the presence or absence of Ta1 on PBMCs from healthy donors (Figure $1 \mathrm{C})$ and patients (Figure 1D; $\mathrm{P}>0.05$ ). Therefore, these results showed that Ta1 treatment was able to increase the $\mathrm{CD}^{+}{ }^{+} \mathrm{CD} 25^{+} \mathrm{Foxp}^{+}$Treg, but not the $\mathrm{CD}^{+}{ }^{+}$Foxp3 ${ }^{+}$Treg differentiation. The effect of Ta1 in terms of CD8 ${ }^{+}$Foxp $^{+}$ Treg expression has not been reported.

Ta1 elevates the levels of the inflammatory cytokines IL-1 $\beta$ and TNF- $\alpha$ in the PBMCs from gastric cancer patients. We used cytokine-FACS analysis to detect the effects of Ta1 on Th1 cytokine production. PBMCs and TILs were stimulated with Ta1 at various concentrations, and the secretion of IL-1 $1 \beta$, IL-2, IL-12 (p70), IFN- $\gamma$, TNF- $\alpha$, and TNF- $\beta$ was subsequently measured. The data in Figure 2, shows that the production of IFN-y in PBMCs from healthy donors was higher than in PBMCs from gastric cancer patients. Treatment with Ta1 at various concentrations resulted in no significant changes of IFN-Y production in PBMCs from either donors or patients $(P>0.05)$. No IFN-y secretion was detected in TILs from patients. Production of IL-1 $\beta$ in PBMCs from healthy donors was lower than in PBMCs from patients. Treatment with $1 \mu \mathrm{g} / \mathrm{mL}$ Ta1 caused an increase to $178 \%$ in the secretion of IL-1 $\beta$ by PBMCs from patients compared to those untreated $(100 \%$; $P<0.05)$. In contrast, $1 \mu \mathrm{g} / \mathrm{mL}$ Ta1 induced secretion of TNF- $\alpha$ to more than $500 \%$ in PBMCs from patients compared to other various treatment conditions. However, Ta1 decreased TNF- $\alpha$ levels in PBMCs from healthy donors under various treatment conditions $(P<0.05)$. The IL-2, IL-12 (p70), and TNF- $\beta$ secretion profiles in PBMCs from patients and donors or TILs from patients were not significantly different under any condition (data not shown).

Ta1 elevates the levels of IL-6 Th2-cytokines in PBMCs. Cytokine profiles were assessed after stimulation with Ta1 and Th2 cytokines (including IL-4, IL-5, IL-6, and IL-10). As shown in Figure 3, Ta1 treatment caused no significant changes in IL-10 secretion by PBMCs from patients and healthy donors $(P>0.05)$. The level of IL-10 was close to $0 \mathrm{pg} / \mathrm{mL}$ in TILs from patients. Ta1 showed no significant changes in secretion of IL-10 in TILs from gastric cancer patients $(P>0.05)$. However, Ta1 at concentrations of 

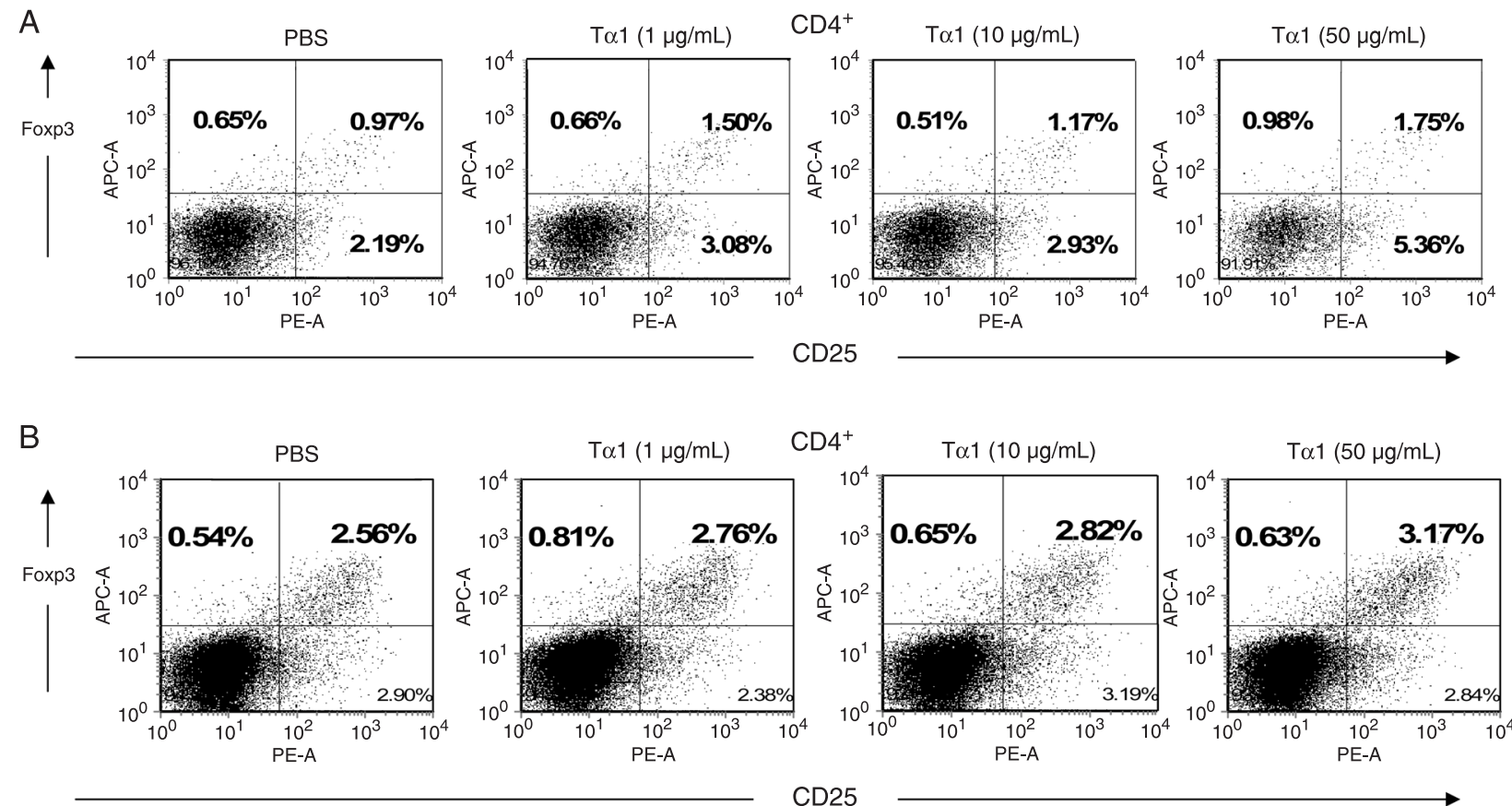

CD25
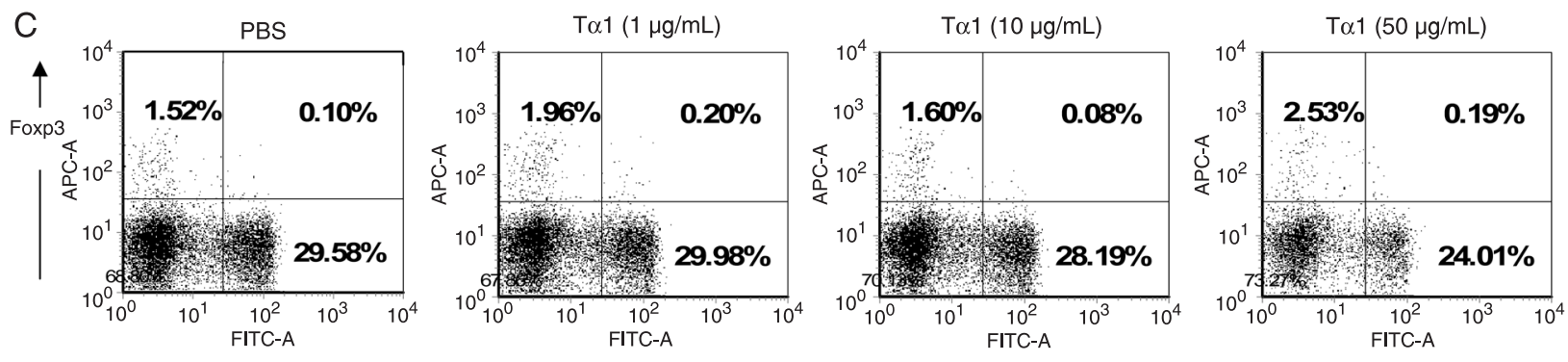

CD8
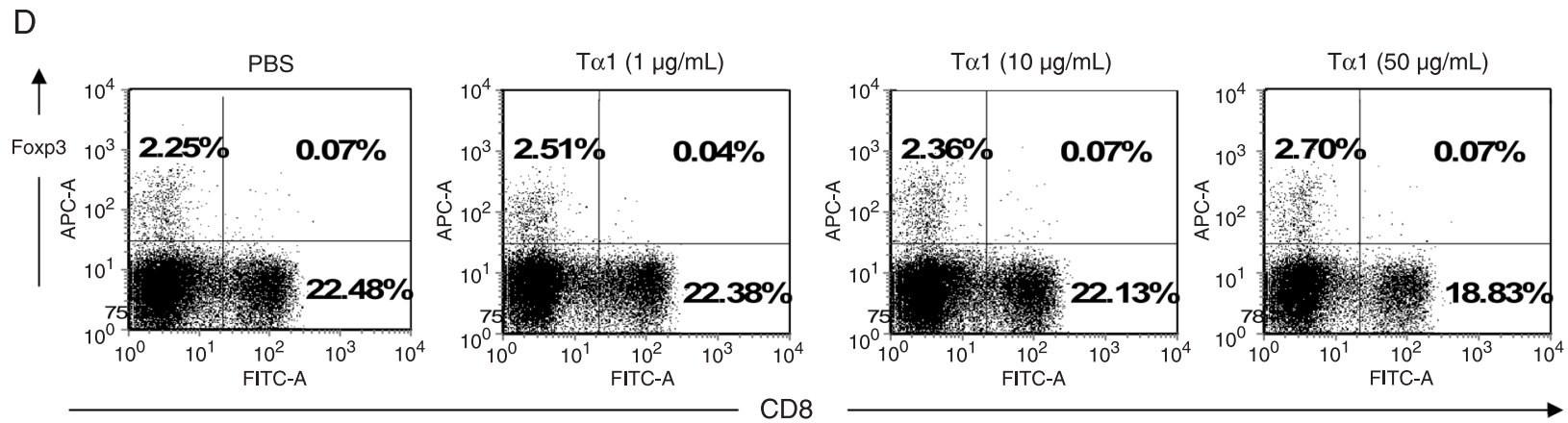

Figure 1. Frequency of $\mathrm{CD} 4^{+}$and $\mathrm{CD} 8^{+}$Tregs in PBMCs from healthy donors and gastric cancer patients upon treatment with $\mathrm{PBS}$ or with 1,10 , or $50 \mu \mathrm{g} / \mathrm{mL}$ Ta1. A, Analysis of the CD4 ${ }^{+} \mathrm{CD} 25^{+} \mathrm{Foxp} 3^{+}$subpopulation of PBMCs from healthy donors and $B$, gastric cancer patients. $C$, Analysis of the $\mathrm{CD} 8^{+}$and $\mathrm{CD} 8^{+} \mathrm{Foxp} 3^{+}$subpopulations of PBMCs from healthy donors and $D$, gastric cancer patients. Tregs $=$ regulatory $\mathrm{T}$ cells; $\mathrm{PBMCs}=$ peripheral blood mononuclear cells . 

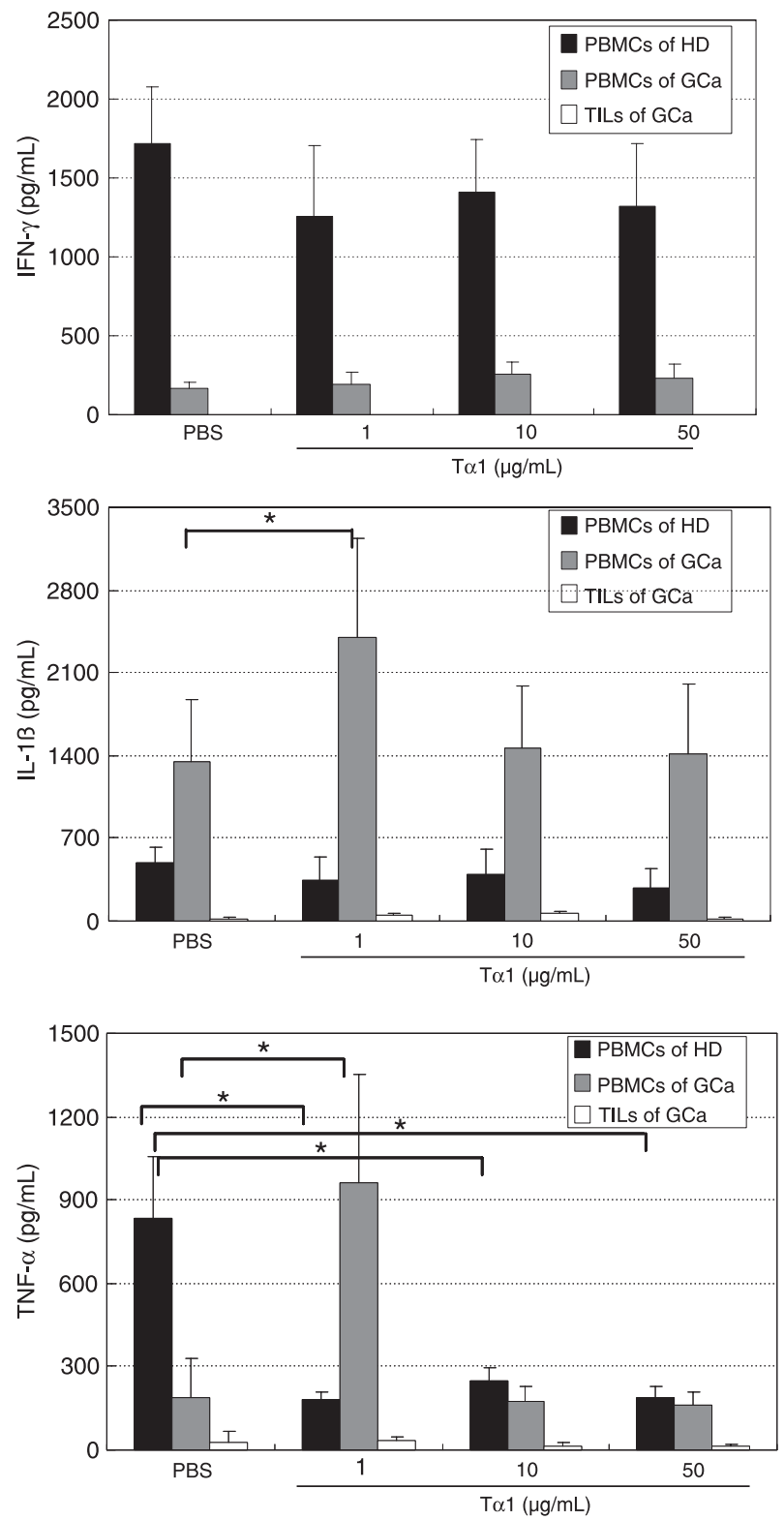

Figure 2. Effect of Ta1 on Th1 cytokine secretion in PBMCs from healthy donors (HD) and gastric cancer patients (GCa) and in TILs from gastric cancer patients. PBMCs and TILs were cultured with PBS or 1,10 , or $50 \mu \mathrm{g} / \mathrm{mL}$ Ta1 for $72 \mathrm{~h}$. The levels of Th1 cytokines were determined using the Bio-Plex cytokine assay. PBMCs = peripheral blood mononuclear cells; TILs = tumorinfiltrating lymphocytes. Data are reported as means \pm SD for 3 separate experiments $(P<0.05, t$-test $)$.

$10 \mu \mathrm{g} / \mathrm{mL}$ increased the secretion of IL- 6 to over $140 \%$ in PBMCs from both healthy donors and patients $(P<0.05)$. The IL-4 and IL-5 secretion levels were undetectable in PBMCs from patients and donors or TILs from patients under the various treatment conditions (data not shown).

Ta1 seems to be inconsistent in inducing the secretion

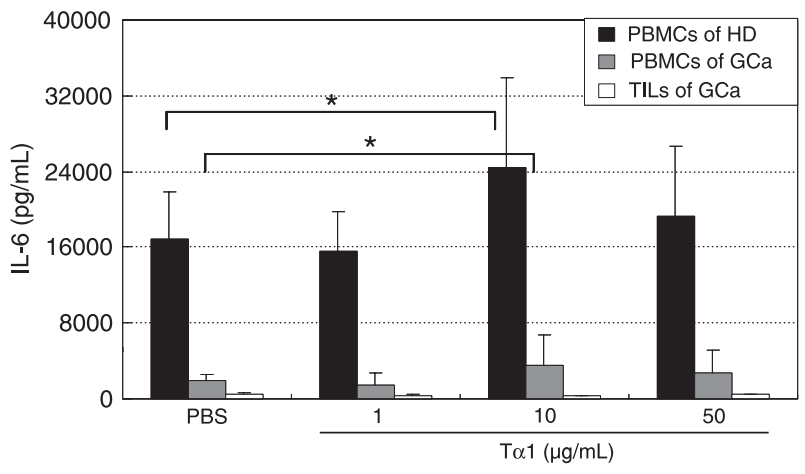

Figure 3. Effect of Ta1 on IL-6 cytokine secretion in PBMCs from healthy donors (HD) and gastric cancer patients (GCa), and in TILs from gastric cancer patients. PBMCs and TILs were cultured with PBS or 1,10 , or $50 \mu \mathrm{g} / \mathrm{mL}$ Ta1 for $72 \mathrm{~h}$. The levels of Th2 cytokines were determined using the Bio-Plex cytokine assay. PBMCs = peripheral blood mononuclear cells; TILs = tumorinfiltrating lymphocytes. Data are reported as means \pm SD for 3 separate experiments $(P<0.05, t$-test $)$.

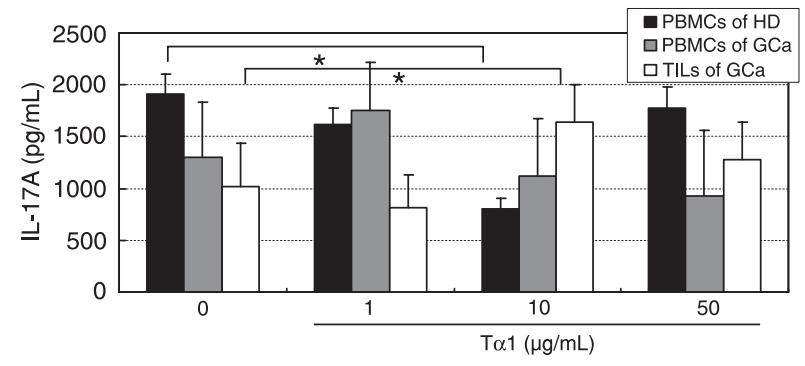

Figure 4. Effect of Ta1 on IL-17A secretion in PBMCs from healthy donors (HD) and gastric cancer patients (GCa) and in TILs from gastric cancer patients. PBMCs and TILs were cultured with PBS or 1,10 , or $50 \mu \mathrm{g} / \mathrm{mL}$ Ta1 for $72 \mathrm{~h}$. Th17A levels were determined by a Bio-Plex cytokine assay. PBMCs = peripheral blood mononuclear cells; TILs = tumor-infiltrating lymphocytes. Data are reported as means $\pm S D$ for 3 separate experiments ( ${ }^{*} P$ $<0.05, t$-test).

of IL-17A. To further confirm the role of Ta1 in Th17 cytokine secretion in PBMCs, IL-17A was examined by flow cytometry analysis. As shown in Figure 4, we found that Ta1 at concentrations of $10 \mu \mathrm{g} / \mathrm{mL}$ decreased IL-17A secretion to $42 \%$ in PBMCs from healthy donors $(P<0.05)$, but there were no significant changes detected in the secretion of IL-17A in PBMCs from patients. IL-17A in TILs from patients was increased by $60 \%$ with $10 \mu \mathrm{g} / \mathrm{mL}$ Ta1, compared to PBS-treated cells $(100 \% ; P<0.05)$. These data suggest that Ta1 might reduce the secretion of IL-17A in cells from healthy subjects. However, Ta1 did not decrease the secretion of IL-17A in PBMCs from gastric cancer patients. $T \alpha 1$, therefore, has an inconsistent function in inducing the secretion of IL-17A. 
Ta1 had no significant effect on T lymphocyte proliferation. To investigate the impact of Ta1 on PBMC proliferative capacity, PBMCs from healthy individuals and patients with gastric cancer were incubated with $T \alpha 1$ at various concentrations. Cell proliferation was measured by the incorporation of $\left[{ }^{3} \mathrm{H}\right]$-thymidine. Ta1 caused no significant change in the proliferative capacity of PBMCs from healthy donors and gastric cancer patients when compared to PBS-treated cells $(P>0.05)$.

\section{Discussion}

Recent studies have demonstrated that some immune cells such as T and NK cells produce specific cytokines, which normally function as suppressors of tumors. Similarly, one of the key elements sought to develop effective tumor immunotherapies is the means by which to induce effective host-derived immune effectors in the tumor microenvironment. These immune cells and cytokines influence tumorigenesis, including growth and metastasis. One of the main mechanisms for immune elimination of tumor cells is the release (exocytosis) of cytolytic granules by cytotoxic $\mathrm{CD}^{+} \mathrm{T}$ lymphocytes (CTLs) and NK cells, a process that is mediated by adequate contact and antigenic recognition of tumor cells. Still another pathway requires the interaction of Fas ligands on the surface of CTLs with Fas molecules present on target tumor cells. However, it has been demonstrated that the interaction between target and NK cells can be prevented by negative signaling, causing NK cells to lose their cytotoxic capacity and to become functionally "exhausted". Moreover, IL-15, IL-2, IFN- $\alpha$, and IL-21 (22) have been demonstrated to promote NK- and $\mathrm{CD} 8^{+} \mathrm{T}$ cellmediated cytotoxicity, to sustain function, and to increase antitumor immunity. Animal studies have shown that Ta1 had no effect on T cell numbers (both $\mathrm{CD}^{+}$and $\mathrm{CD} 8^{+}$), and therefore, had no effect on $\mathrm{T}$ lymphocyte proliferation (23). Therefore, our in vitro data are consistent with this finding.

Control of tumor growth depends on the clearance of the tumor by antitumor immune responses, and on effectively abrogating immunological unresponsiveness. CD $4^{+} \mathrm{CD} 25^{+}$ Tregs have been shown to functionally contribute to the maintenance of immune tolerance and prevention of autoimmunity. However, Tregs can also prevent host antitumor immune responses. Removal of $\mathrm{CD} 4^{+} \mathrm{CD} 25^{+}$Tregs has been shown to be effective for boosting tumor-specific immunity in vivo in a mouse model (24). In humans, tumor Tregs appear to exert immunosuppressive effects on the proliferation and activation of immune effector cells, increasing the growth of tumors in vivo and reducing the survival rate of individuals with ovarian cancer (12). CD $4^{+} \mathrm{CD} 25^{+}$Tregs are one of the active weapons utilized by tumors to circumvent host immunity. When Tregs were incubated together with $\mathrm{CD}^{2} 6^{+} \mathrm{NK}$ cells, the NK cells lost their cytotoxic function (5). Depletion of Tregs in tumors can evoke specific host immunity, representing an attractive anticancer strategy. Conversely, it could be beneficial for the control of autoimmune disorders. In a CLP septic mouse model, Ta1 was found to decrease the percentage of $\mathrm{CD} 4^{+} \mathrm{CD} 25^{+} \mathrm{Foxp} 3^{+}$ $\mathrm{T}$ lymphocytes (25). The present study revealed that Ta1 can increase levels of Tregs in PBMCs from patients with gastric cancer, suggesting that Ta1 could increase the risk of patients to develop tumor immunotolerance.

Other immune effectors, such as cytokines and chemokines, are known to play either positive or negative roles in cancer immunity in the tumor microenvironment. Much attention has been given recently to defining the cytokine secretion profiles of immune cells since they are involved in antitumor immunity. IL- 6 has been documented as a pleiotropic cytokine, a potent pro-angiogenic molecule that stimulates $\mathrm{T}$ lymphocyte proliferation and differentiation. Furthermore, IL-6 is also thought to be a tumor cell growth factor for tumor cell proliferation, which contributes to drug resistance, cachexia, and other problems (26).

In addition, IL-10 can act as an inhibitory cytokine, playing an important role in the regulation of $\mathrm{T}$ cell responses and tumor-immune escape effects (27). IFN- $\alpha$ and its receptor, IFNR- $\alpha$, are involved in inhibiting angiogenesis in tumor rejection mediated by $\mathrm{CD}^{+}$or $\mathrm{CD}^{+} \mathrm{T}$ cells $(28,29)$. It has been reported that IL-4 levels were significantly higher in HBV patients, while IL-10 levels were significantly lower with Ta1 treatment compared to controls (20). Ta1 was documented to increase the secretion of IL-10 by $53 \%$ in Panc-1 pancreatic cells. However, Ta1 did not cause an increase of IL-10 in BxPC-3 cells, another pancreatic cancer cell line (30). These data suggest that the Ta1-stimulating effect on the secretion of IL-10 is variable.

Previous reports have indicated that $\mathrm{T \alpha} 1$ did not result in any significant differences in IFN-y concentrations between HBV patients and controls at baseline conditions. However, Ta1 significantly increased the concentration of IL-2 of HBV-infected patients compared with healthy donors (20). Some reports have indicated that treatment of Ta1 increased serum IL-1 $\beta$ levels, but not IL-2 levels, in rats (31). Our findings suggest that Ta1 at some concentration can elevate levels of the inflammatory cytokines IL-1 $\beta$ and TNF- $\alpha$ in the PBMCs from patients. Previous research has suggested that the elevation of serum IL-6 and TNF- $\alpha$ in patients with prostate cancer correlated with poor survival (32). We conclude that Ta1 can elevate the levels of IL-1 $\beta$ and TNF- $\alpha$ in the PBMCs from patients, providing preclinical evidence supporting the efficacy of a Ta1 blockade against malignant gastric carcinoma.

Cytokine IL-17 has been shown to have a potent effect on tumor cell proliferation and angiogenesis (33). Similarly, treatment with Ta1 was also found to stimulate secretion of IL-17 in Panc-1 and BxPC-3 cells (30).

Our data show that Ta1 at different concentrations can increase the levels of Tregs and the production of the inflammatory cytokines TNF- $\alpha, \mathrm{IL}-1 \beta$, and IL- 6 in the 
PBMCs, suggesting that different qualitative effects of Ta1 may depend on the concentration. In addition, we found that $\mathrm{Ta} 1$ at a concentration of $10 \mu \mathrm{g} / \mathrm{mL}$ decreased the secretion of IL-17A in PBMCs from healthy donors. However, Ta1 did not modify the secretion of IL-17A in PBMCs from gastric cancer patients. These data indicate that the effect of Ta1 on the regulation of IL-17A secretion is also inconsistent. Taken together, our results suggest that the effect of Ta1 on regulation of Th17 cytokine secretion is inconsistent. Our data suggest that the effect of Ta1 on the up-regulation of Tregs in PBMCs from gastric patients is

\section{References}

1. Parkin DM, Bray F, Ferlay J, Pisani P. Global cancer statistics, 2002. CA Cancer J Clin 2005; 55: 74-108.

2. Sah BK, Chen MM, Yan M, Zhu ZG. Reoperation for early postoperative complications after gastric cancer surgery in a Chinese hospital. World J Gastroenterol 2010; 16: 98-103.

3. Boku N, Yamamoto S, Fukuda H, Shirao K, Doi T, Sawaki A, et al. Fluorouracil versus combination of irinotecan plus cisplatin versus S-1 in metastatic gastric cancer: a randomised phase 3 study. Lancet Oncol 2009; 10: 1063-1069.

4. Curiel TJ, Wei S, Dong H, Alvarez X, Cheng P, Mottram P, et al. Blockade of B7-H1 improves myeloid dendritic cellmediated antitumor immunity. Nat Med 2003; 9: 562-567.

5. Wolf AM, Wolf D, Steurer M, Gastl G, Gunsilius E, GrubeckLoebenstein B. Increase of regulatory T cells in the peripheral blood of cancer patients. Clin Cancer Res 2003; 9: 606-612.

6. Bhairavabhotla RK, Verm V, Tongaonkar H, Shastri S, Dinshaw K, Chiplunkar S. Role of IL-10 in immune suppression in cervical cancer. Indian J Biochem Biophys 2007; 44: 350356.

7. Toutirais $\mathrm{O}$, Chartier $\mathrm{P}$, Dubois $\mathrm{D}$, Bouet $\mathrm{F}$, Leveque $\mathrm{J}$, Catros-Quemener V, et al. Constitutive expression of TGFbeta1, interleukin- 6 and interleukin- 8 by tumor cells as a major component of immune escape in human ovarian carcinoma. Eur Cytokine Netw 2003; 14: 246-255.

8. Singh $H$, Jain M, Sachan R, Mittal B. Association of TNFA $(-308 \mathrm{G}>\mathrm{A})$ and IL-10 (-819C>T) promoter polymorphisms with risk of cervical cancer. Int J Gynecol Cancer 2009; 19: 1190-1194

9. Wang L, Yi T, Kortylewski M, Pardoll DM, Zeng D, Yu H. IL-17 can promote tumor growth through an IL-6-Stat3 signaling pathway. J Exp Med 2009; 206: 1457-1464.

10. He D, Li H, Yusuf N, Elmets CA, Li J, Mountz JD, et al. IL-17 promotes tumor development through the induction of tumor promoting microenvironments at tumor sites and myeloid-derived suppressor cells. J Immunol 2010; 184: 2281-2288.

11. Khazaie $\mathrm{K}$, von Boehmer $\mathrm{H}$. The impact of $\mathrm{CD} 4+\mathrm{CD} 25+$ Treg on tumor specific CD8+ T cell cytotoxicity and cancer. Semin Cancer Biol 2006; 16: 124-136.

12. Curiel TJ, Coukos G, Zou L, Alvarez X, Cheng P, Mottram $P$, et al. Specific recruitment of regulatory $T$ cells in ovarian carcinoma fosters immune privilege and predicts reduced survival. Nat Med 2004; 10: 942-949. confirmed with the changes of secretion of TNF- $\alpha, I L-1 \beta$, and IL-6. While the data are suggestive, we recognize that some observed changes are small, and sometimes not statistically significant. Therefore, these results need to be confirmed in larger studies.

\section{Acknowledgments}

Research supported by the National Natural Science Foundation of China (\#30972716).
13. Haritos AA, Goodall GJ, Horecker BL. Prothymosin alpha: isolation and properties of the major immunoreactive form of thymosin alpha 1 in rat thymus. Proc Natl Acad Sci U S A 1984; 81: 1008-1011.

14. Hsia J, Sarin N, Oliver JH, Goldstein AL. Aspirin and thymosin increase interleukin-2 and interferon-gamma production by human peripheral blood lymphocytes. Immunopharmacology 1989; 17: 167-173.

15. Shrivastava P, Singh SM, Singh N. Effect of thymosin alpha 1 on the antitumor activity of tumor-associated macrophagederived dendritic cells. J Biomed Sci 2004; 11: 623-630.

16. Shrivastava P, Singh SM, Singh N. Antitumor activation of peritoneal macrophages by thymosin alpha-1. Cancer Invest 2005; 23: 316-322.

17. Goldstein AL, Goldstein AL. From lab to bedside: emerging clinical applications of thymosin alpha 1. Expert Opin Biol Ther 2009; 9: 593-608.

18. Roman S, Petrusca D, Moldovan I, Paraoan M, Petrescu A, Damian D, et al. Evaluation of apoptosis of tumor and of apparently normal cells in human renal carcinoma. Immunol Lett 1999; 67: 15-22.

19. Shimizu Y, Weidmann E, Iwatsuki S, Herberman RB, Whiteside TL. Characterization of human autotumor-reactive T-cell clones obtained from tumor-infiltrating lymphocytes in liver metastasis of gastric carcinoma. Cancer Res 1991; 51: 6153-6162.

20. Loggi E, Gramenzi A, Margotti M, Cursaro C, Galli S, Vitale $\mathrm{G}$, et al. In vitro effect of thymosin-alpha1 and interferonalpha on Th1 and Th2 cytokine synthesis in patients with eAg-negative chronic hepatitis B. J Viral Hepat 2008; 15: 442-448.

21. Chadwick D, Pido-Lopez J, Pires A, Imami N, Gotch F, Villacian JS, et al. A pilot study of the safety and efficacy of thymosin alpha 1 in augmenting immune reconstitution in HIV-infected patients with low CD4 counts taking highly active antiretroviral therapy. Clin Exp Immunol 2003; 134: 477-481.

22. Yi JS, Du M, ZajacAJ. A vital role for interleukin-21 in the control of a chronic viral infection. Science 2009; 324: 1572-1576.

23. Hadden JW, Verastegui E, Hadden E. IRX-2 and thymosin alpha1 (Zadaxin) increase $T$ lymphocytes in $T$ lymphocytopenic mice and humans. Ann N Y Acad Sci 2007; 1112: 245-255.

24. Shimizu J, Yamazaki S, Sakaguchi S. Induction of tumor 
immunity by removing CD25+CD4+ T cells: a common basis between tumor immunity and autoimmunity. $J$ Immunol 1999; 163: 5211-5218.

25. Wan J, Shan Y, Shan H, Li G, Wang T, Guan J, et al. Thymosin-alpha1 promotes the apoptosis of regulatory $T$ cells and survival rate in septic mice. Front Biosci 2011; 17: 3004-3013.

26. Zaki MH, Nemeth JA, Trikha M. CNTO 328, a monoclonal antibody to IL-6, inhibits human tumor-induced cachexia in nude mice. Int J Cancer 2004; 111: 592-595.

27. Urosevic M, Dummer R. HLA-G and IL-10 expression in human cancer - different stories with the same message. Semin Cancer Biol 2003; 13: 337-342.

28. Qin Z, Blankenstein T. CD4+ T cell-mediated tumor rejection involves inhibition of angiogenesis that is dependent on IFN gamma receptor expression by nonhematopoietic cells. Immunity 2000; 12: 677-686.

29. Qin Z, Schwartzkopff J, Pradera F, Kammertoens T, Se- liger $\mathrm{B}$, Pircher $\mathrm{H}$, et al. A critical requirement of interferon gamma-mediated angiostasis for tumor rejection by CD8+ T cells. Cancer Res 2003; 63: 4095-4100.

30. Li M, Feurino LW, Li F, Wang H, Zhai Q, Fisher WE, et al. Thymosinalpha1 stimulates cell proliferation by activating ERK1/2, JNK, and increasing cytokine secretion in human pancreatic cancer cells. Cancer Lett 2007; 248: 58-67.

31. Yao W, Zhu Q, Yuan Y, Qiao M, Zhang Y, Zhai Z. Thymosin alpha 1 improves severe acute pancreatitis in rats via regulation of peripheral T cell number and cytokine serum level. $J$ Gastroenterol Hepatol 2007; 22: 1866-1871.

32. Michalaki V, Syrigos K, Charles P, Waxman J. Serum levels of IL-6 and TNF-alpha correlate with clinicopathological features and patient survival in patients with prostate cancer. $\mathrm{Br}$ J Cancer 2004; 90: 2312-2316.

33. Numasaki M, Fukushi J, Ono M, Narula SK, Zavodny PJ, Kudo $\mathrm{T}$, et al. Interleukin-17 promotes angiogenesis and tumor growth. Blood 2003; 101: 2620-2627. 